\title{
DIE PARLAMENTSWAHLEN IN THAILAND (1969)
}

I. Früher als allgemein erwartet fanden am 10. Februar 1969 in Thailand die Wahlen zum Abgeordnetenhaus statt. Gemäß Artikel 180 der Verfassung 2511 sollte die Wahl der Mitglieder zum Abgeordnetenhaus binnen 240 Tagen, gerechnet vom Inkrafttreten der Verfassung an, stattfinden. Die Verfassung trat am 20. Juni 1968 in Kraft. Die Wahl fand somit nach 235 Tagen statt, d. h. verfassungskonform. Die Militärregierung unter Thanom Kitičakọn widerstand der Versuchung, von der Möglichkeit des Artikel 180, die Frist auf unbestimmte Zeit zu verlängern, Gebrauch zu machen.

Verschiedene Gründe mögen die Militärs bewogen haben, das Wagnis und für sie eventuell risikoreiche Unternehmen der verfassungsgemäßen Wahl einzugehen: Demokratie vermag sich außenpolitisch besser zu verkaufen als eine Militärdiktatur. Vielleicht mag die Einsicht, $\mathrm{da} ß$ es an der Zeit war, der Bangkoker Opposition nachzugeben, einer der Gründe gewesen sein. Sicherlich werden auch die beträchtlichen Erfolge der Regierung bei der Verbesserung der Wirtschaftslage und damit ein zu vermutender größerer Rückhalt bei der Bevölkerung die Erörterungen vor der Wahl mitbestimmt haben. Man sollte ferner nicht die politische Klugheit der thailändischen Regierung unterschätzen. Die Einsicht, daß offene, jedermann zugängliche Debatten und Mitheranziehung zur Verantwortung geeignetere Mittel sind, kommunistischer Beeinflussung $\mathrm{zu}$ begegnen als obrigkeitsstaatliche-patriarchalische Gewaltmaßnahme ist gewiß vorhanden. Und möglicherweise spielte auch das Kalkül eine Rolle, daß das Königreich Thailand in der Zukunft vor politischen Entscheidungen stehen könnte, die alleine zu verantworten die Militärregierung nicht auf sich nehmen wollte.

II. Nach Artikel 83 der Verfassung 2511 richtet sich die Anzahl der Abgeordneten, die in den 71 Provinzen (Čangwat) Thailands zu wählen sind, nach deren Einwohnerzahl. Auf 150000 Einwohner ist ein Abgeordneter zu wählen. Ein Bruchteil dieser Zahl, der 75000 überschreitet, berechtigt gleichfalls zur Wahl eines Abgeordneten. Bei einer Gesamtbevölkerung von $32468653^{1}$ waren 219 Abgeordnete $\mathrm{zu}$ wählen. In der Provinz Bangkok/Thonburi mit 2275684 Einwohnern davon allein 21, in manchen entlegenen, bevölkerungsarmen Grenzprovinzen nur ein Abgeordneter.

Als Wahlbezirke wurden in den Provinzen die Tambon genannten Verwaltungseinheiten zugrunde gelegt ${ }^{2}$. Die Provinzgouverneure waren jedoch ermächtigt, bevölkerungsstarke Tambon mit mehr als 2000 Wahlberechtigten zu unterteilen.

Gewählt war derjenige Kandidat, der die größte Zahl der abgegebenen Stimmen erhielt. Konnten in einer Provinz mehrere Abgeordnete gewählt werden, so galten die mit der nächsthöheren Stimmenzahl als gewählt.

Die Qualifikation zum aktiven Wahlrecht richtete sich nach den Bestimmungen der Artikel 84 bis 86, die zum passiven Wahlrecht nach den Bestimmungen der Artikel 87 bis 89 der Verfassung $2511^{3}$.

Der äußere Verlauf der Wahl, auch der der vorbereitenden Kandidatenwerbung, verlief nach übereinstimmenden Presseberichten ${ }^{4}$ ruhig und geordnet. Tätlichkeiten oder persönliche Verunglimpfungen unterblieben im allgemeinen ${ }^{5}$, wo-

1 Entnommen einer vom thailändischen Innenministerium anläßlich der Wahl herausgegebenen Broschüre nGeneral Election in Thailand 10 February 1969\%, published by the Departement of Loeul Administration,

2 Tambon: eine aus mehreren Gemeinden bestehende Verwaltungseinheit.

3 S. Wenk. Die neue Verfassung Thailands, Verfassung und Recht in Ubersee, 1. Heft 1969, S. $96 \mathrm{f}$.

4 S. u. a. Bangkok Post vom 12. 2. 69; Bangkok World vom 12. 2. 69. osthailand), wo zwei Wahloffizielle von kommunistischen Guerillas erschossen wurden, und bei Narathiwat 
durch sich die diesjährige Wahl wohltuend von den in den Jahren 1946 und 1957 stattgefundenen unterschied. Auf die teilweise in deutlicher Form vorgetragene Kritik seitens der Demokratischen Partei an der Militärregierung reagierte diese gelassen.

III. Um die 219 Sitze im Abgeordnetenhaus bewarben sich im ganzen Land 1254 Kandidaten, die einer der elf zugelassenen Parteien angehörten oder als unabhängige Kandidaten auftraten.

Die Parteien Thailands unterscheiden sich sowohl ihrer Struktur als auch ihrer Zielsetzung nach wesentlich von den westlichen politischen Parteien. Ihr Ausgangs-, ihr Kristallisationspunkt ist im allgemeinen nicht eine bestimmte Ideologie, sondern eine profilierte Persönlichkeit des politischen Lebens. Mit dem Tod oder dem Sturz des Parteiführers endet meistens auch die Partei. Sie verfügen kaum über eine Organisation, einen Apparat. Deutlicher als dies in westlichen Parteien zutage tritt, sind sie Interessengemeinschaften einer bestimmten Bevölkerungsgruppe, die sehr konkrete irdische Ziele zu erreichen anstreben.

Die Chance, gewählt zu werden, hat, besonders in den ländlichen Provinzialwahlkreisen, nur eine der Bevölkerung bekannte Persönlichkeit. Daraus ist u. a. die große Anzahl der unabhängigen Abgeordneten zu erklären.

Presseberichten nach ist ein neues Parteiengesetz geplant, das bezwecken soll, künftig nur noch Kandidaten, die einer der zugelassenen Parteien angehören, zu den Wahlen zuzulassen. Das Mandat eines Abgeordneten soll erlöschen, wenn dieser die Partei, als deren Kandidat er gewählt wurde, verläßt. Dieser Plan zielt wohl darauf ab, die Stellung der sog. „Regierungspartei“ des Ministerpräsidenten zu stärken.

Im bisherigen politischen Leben Thailands haben die Parteien eine relativ bescheidene Rolle gespielt. Zu keiner Zeit war eine Partei so stark, daß sie der Volksvertretung ihren Willen aufzwingen konnte, d. h. daß die zu fassenden Beschlüsse im Parteivorstand festgelegt werden konnten. Soweit eine Partei bisher maßgebend an der Mitgestaltung der Politik Thailands beteiligt war, war es stets nur ein relativ kurzes Gastspiel und stets nur in der Rolle eines Lückenbüßers für die Militärregierung. Als Beispiele seien genannt: nach dem Ende des zweiten Weltkrieges, als sich die Militärregierung unter Phibun Songkhram des Bündnisses mit den Japanern wegen den Alliierten gegenüber als Verhandlungspartner diskreditiert hatte ${ }^{6}$; im Jahre 1955, als der das Ende seiner Ära wohl ahnende Phibun Songkhram sich vor rivalisierenden Militärgruppen mit Hilfe politischer Parteien absichern wollte?

Vergegenwärtigt man sich die Streichung des Artikels 61 der Verfassung von 1949 , so könnte dies vielleicht als ein Indiz dafür angesehen werden, daß sich eine neue Entwicklung anbahnt, d. h. daß die Parteien in Zukunft - mit Unterstützung der Militärfaktion - ein integrierender Bestandteil des politischen Lebens werden ${ }^{8}$.

Im einzelnen bewarben sich bei den diesjährigen Wahlen Kandidaten der folgenden elf Parteien ${ }^{9}$ :

1. Saha Pracha Thai, „Vereinigtes Thai-Volk“. Sie ist auch als „Regierungspartei“ bekannt. Vorsitzender ist der langjährige Ministerpräsident Thanom Kitičakon, sein Stellvertreter der Innenminister Prapat.

Saha Pracha Thai ist die Nachfolgeorganisation der sog. „Revolutionspartei“ des verstorbenen Premiers Sarit Thanarat. Fast alle Regierungsmitglieder gehören ihr an.

(Südthailand, nahe der malaiischen Grenze), wo „die Polizei zwei von 60 angreifenden kommunistischen Terroristen " erschoß; item Phim Thai vom 11. 2. 69.

6 S. Wenk, Die Verfassungen Thailands, FfM. u. Berlin 1964, S. 11, dort weitere Literaturnachweise.

7 Wenk, op. cit., S. 13.

8 S. Wenk, op. cit. Anm. 3, S. 85

9 Nach op. cit. Anm. 1, S. 9 f. 
Das jeweilige Programm dieser Partei mag aus den Regierungserklärungen ersehen werden. Das bedeutet, daß jenes nicht ideologisch fixiert ist, sondern sich pragmatisch an den Erfordernissen der politischen Situation ausrichtet. Als "gemäßigt konservativ" könnte man die Richtung der Regierungspartei charakterisieren. Außenpolitisch wird nach wie vor eine enge Bindung an die USA angestrebt sowie an westlich-antikommunistisch ausgerichtete Allianzen wie die SEATO. Innenpolitisch steht die wirtschaftliche und bildungsmäßige Entwicklung des Landes im Vordergrund. Man glaubt, eine die Masse der Bevölkerung befriedigende Entwicklung auf evolutionärem Wege herbeiführen zu können. Ferner ist. die Bekämpfung kommunistischer Infiltrationsversuche ein erklärter und rigoros gehandhabter Programmpunkt.

2. Prachathipatai, „Demokratische Partei“. Unter dem Vorsitz des früheren Ministerpräsidenten Seni Pramot ist die Prachathipatai d i e Oppositionspartei. Sie ist bürgerlich ausgerichtet. Gegründet wurde sie im Jahre 1946 von Khuang Aphaiwong, der von 1944 bis 1948 dreimal Ministerpräsident war. Trotz des im Jahre 1958 von Sarit erlassenen Parteienverbots gelang es, die Organisation dieser Partei einigermaßen intakt zu halten, wohl vor allem deswegen, weil die Unzufriedenheit der demokratisch eingestellten Opposition während des zehnjährigen Parteienverbots hier ein Sammelbecken fand.

Nach dem Tode des in der Bevölkerung hohes Ansehen, ja Verehrung genießenden Khuang Aphaiwong am 15. 3. 1968, übernahm Seni Pramot, eine Persönlichkeit von überragender Intelligenz, die Führung der Partei. Der neue Vorsitzende war während des zweiten Weltkrieges Botschafter Thailands in Washington. 1941 weigerte er sich, die unter japanischem Druck zustandegekommene Kriegserklärung Thailands an die USA zu überreichen. Er sagte sich am 16. 12. 1941 von der Regierung Phibun Songkhram los und gründete in den USA das „Free Thai Movement“. Im Jahre 1945, nach seiner Rückkehr, wurde er Ministerpräsident, blieb dies jedoch nur vier Monate bis zu den Wahlen am 6. 1.1946.

Das Programm der Demokratischen Partei war unter Khuang Aphaiwong innenpolitisch von einer konservativen, außenpolitisch von einer leicht neutralistischen Richtung getragen. Doch es scheint so, daß sich heute mehr eine fortschrittlich-liberale Richtung durchzusetzen beginnt. Populär ist der Programmpunkt „Bekämpfung der Korruption“. Auch die Regierungspartei fordert dies, doch wird deren Forderung von der Bevölkerung mit Skepsis aufgenommen.

Der leicht neutralistische Kurs in der Außenpolitik bedeutet keinesfalls eine Aufgabe der engen, freundschaftlichen Bindungen an die USA, aber doch ein gewisses Abrücken von der einseitigen Bindung. Vor allem bedeutet „leicht neutralistisch" für die Demokratische Partei die Möglichkeit, China diplomatisch anzuerkennen. Aus den Reden, Interviews und Presseartikeln thailändischer Politiker während der letzten Jahre ${ }^{\mathbf{1 0}}$ ist $\mathrm{zu}$ erkennen, daß solche Überlegungen auch bei der herrschenden Militärgruppe angestellt werden. Das Ende des Krieges in Vietnam und damit ein weniger ausgeprägtes Engagement der USA in Südostasien wird ohne Zweifel eine gewisse Neuorientierung der thailändischen Politik herbeiführen.

Für das weitere Schicksal der Prachathipatai ist die Auswirkung ihres Programmpunktes, die Vorherrschaft der Militärs auszuschalten, von maßgebender Bedeutung. Dieses Ziel findet die Unterstützung der Mehrheit der Bevölkerung. Aber es ist doch zweifelhaft, ob es der Partei gelingt, auch in provinziellen

10 Der Verf. stützt sich hier auf eigene Wahrnehmungen und Kenntnisse aus den letzten Jahren; doch s. auch, z. B., die Außerung des thailändischen Außenministers Khoman in der Parlamentsdebatte vom 24. 3. d. J. (laut "Presseveröffentlichung Nr. 9/69 der Royal Thai Embassy“, Bad Godesberg): "Der Außenminister bestätigte gestern, daß Thailand nicht von den Grundprinzipien seiner Regierung abgehen werde, daß es aber für offene Gespräche mit dem kommunistischen China bereit sei . . “ und weiter: "Thailands Außenpolitik sei immer unabhängig gewesen . . . 
Wahlkreisen eine bedeutende Anhängerschaft zu gewinnen und damit die Aussicht, die Regierungsgewalt zu übernehmen. Nicht ausgeschlossen werden kann allerdings, daß zu gegebener Zeit ein Teil der Armeeführung dem in der Bevölkerung höchst unbeliebten Innenminister, Armeebefehlshaber, Polizeibefehlshaber und 1. stellvertretendem Premier Prapat die Unterstützung versagt und damit auch den Ministerpräsidenten fallen läßt. Dann wäre - möglicherweise - der Weg für eine vom Militär geduldete Zivilregierung unter Seni Pramot frei.

3. Näu Ruom Sethakon, die "Vereinigte Wirtschaftsfront", hat von allen thailändischen Parteien das am weitesten nach links gerichtete Programm. Es fordert u. a. Staatssozialismus, hohe Besteuerung der Vermögen, außenpolitische Orientierung nach China, Ablehnung der SEATO und des Bündnisses mit den USA. Der Vorsitzende, Thep Chotinuchit, bezeichnete die Partei jedoch als "nicht kommunistisch"11.

Die übrigen Parteien haben nur lokalen Charakter oder sind, wie die Bauernparteien, berufliche Interessengemeinschaften. Ihre Programme bestehen meistens nur aus Gemeinplätzen, wie z. B. die drei Thesen der Isaratham (1) Erhaltung der konstitutionellen Monarchie; (2) Wohlergehen des Volkes; (3) Moral über alles. Daß einige dieser Parteien gleichwohl Sitze im Abgeordnetenhaus erlangten, verdanken sie ihren Vorsitzenden, die örtliche Situationen und Stimmungen auszunutzen verstanden.

Der Vollständigkeit halber seien die weiteren sieben. Parteien hier kurz aufgeführt.

4. Näu Prachathipatai, „Demokratische Linie“.

5. Prachachon, „Volkspartei“.

6. Samachip Chui Chau Na, „Bauernhilfspartei“.

7. Räng Ngan, „Arbeitspartei“.

8. Seri Prachathipatai, „Freie Demokraten“.

9. Chau. Na Chau Rai, „Bauernpartei“.

10. Isaratham, „Freie Demokraten“.

11. Pracha Patana, „Fortschrittliche Volkspartei“.

Ein Teil der kleineren Parteien löste sich bald nach der für sie erfolglosen Wahl wieder auf.

IV. Das amtliche Wahlergebnis sieht wie folgt aus:

1. Saha Pracha Thai

\begin{tabular}{cc} 
Sitze & in Prozenten \\
75 & 34,20 \\
72 & 32,93 \\
57 & 26,00 \\
7 & 3,20 \\
4 & 1,83 \\
2 & 0,92 \\
1 & 0,46 \\
1 & 0,46 \\
\hline 219 & 100,00
\end{tabular}

2. Unabhängige Abgeordnete

3. Prachathipatai

4. Näu Prachathipatai

5. Näu Ruom Sethakhon

6. Prachachon

7. Samachip Chui Chau $\mathrm{Na}$

8. Isaratham

Den oben unter 7. und 9. bis 11. aufgezählten Parteien gelang es nicht, einen Kandidaten durchzubringen.

Die Wahlbeteiligung betrug in den Provinzen durchschnittlich 50 Prozent, in manchen sogar bis zu 72 Prozent, in Bangkok jedoch 34 Prozent. Ermutigend ist hierbei vor allem die im Vergleich zu früheren Wahlen erheblich stärkere Beteiligung der ländlichen Bevölkerung. 
Das Wahlergebnis bietet einige Uberraschungen: 1. das relativ schlechte Ergebnis für die "Regierungspartei“ der Militärs; 2. die hohe Zahl von unabhängigen Abgeordneten; 3. die totale Niederlage der "Regierungspartei“ in der Hauptstadt; 4. die immerhin vier Abgeordneten der Näu Ruom Sethakon in dem streng antikommunistisch ausgerichteten Thailand.

Für die amtierende Militärregierung wird das Ergebnis eine Enttäuschung gewesen sein. Der Ministerpräsident hatte zuversichtlich die Erringung der absoluten Mehrheit für die Saha Pracha Thai vorausgesagt. In der Hauptstadt Bangkok/ Thonburi fielen sämtliche 21 Sitze an die Demokratische Partei Seni Pramots. Wenn die Kapitale auch von jeher der Regierung gegenüber oppositionell eingestellt war, so hatte doch niemand eine Niederlage diesen Ausmaßes für die Saha Pracha Thai erwartet. Es bliebe freilich zu untersuchen, wie die geringe Wahlbeteiligung in Bangkok zu erklären ist.

Andererseits vermag die Militärfaktion für sich als einen beachtlichen Erfolg das gute Abschneiden in den allgemein links-neutralistisch eingestellten Nordostprovinzen zu verbuchen. Die gezielten Maßnahmen zur Verbesserung der Infrastruktur in diesem bisher vernachlässigten Landesteil scheinen Früchte zu tragen.

Die hohe Anzahl der unabhängigen Abgeordneten kann aus der Struktur der politischen Parteien in Thailand erklärt werden. ${ }^{12}$ Dem Hörensagen und Andeutungen in der Presse nach sind etwa 20 bis $30 \mathrm{zu}$ dieser Gruppe gehörende Abgeordnete General Prapat verpflichtet.13 Es sollen die Mitglieder der 1968 gegründeten "Anti-Communist Free People's League of Thailand“ sein. Dem Programm nach war das Ziel dieser Liga die Bekämpfung des Kommunismus. Es ist jedoch bekannt, daß diese Organisation von Vertrauensleuten Prapats begründet wurde und dazu diente, zu dessen Gunsten die Wahl vorzubereiten. Da man sich an das damals noch bestehende Parteienverbot zu halten hatte, gründete man eine „Liga“. Nach einer anderen Äußerung sind 44 „unabhängige“ Abeordnete Mitglieder der Saha Pracha Thai. ${ }^{14}$

Ein sicheres Indiz dafür, daß die Wahlen - von Manipulationen beschränkten Umfangs abgesehen - dem Gesetz entsprechend stattfanden, sind die vier Abgeordneten der Näu Ruom Sethakon. Ihr Programm ist nur in Nuancen von einem kommunistischen Parteiprogramm unterschieden. ${ }^{15}$. Im allgemeinen neigt der Thai nicht zu radikalen Ansichten, dogmatisch fixierte Ideologien entsprechen nicht seiner Weltanschauung. Es bleibt abzuwarten, ob die vier Abgeordneten der Näu Ruom Sethakon nur Vorboten einer wachsenden Wählergemeinschaft oder die Vertreter einer radikalen Minderheit sind. Der Parteivorsitzende Chotinuchit wurde im Wahlkreis Sisaket, im gefährdeten Ostthailand, nicht gewählt.

V. Die Demokratische Partei äußerte sich zufrieden über den Ausgang der Wahlen. Seni Pramot ließ wenige Tage nach der Wahl verlauten, daß die Abgeordneten der Prachathipatai eine „konstruktive Opposition“ betreiben würden ${ }^{16}$. Sie seien, so glaube er persönlich, gewählt worden, weil die Wähler mit der bisherigen Regierung nicht einverstanden gewesen seien und sich eine wirksame Opposition wünschten. Doch gleichzeitig äußerten Mitglieder der Faktion der Unabhängigen ${ }^{17}$, daß wohl der größere Teil der Abgeordneten die Regierungspartei unterstützen würde.

Gemäß Artikel 181 Abs. II der Verfassung endet die Amtszeit einer Regierung, „wenn die Mitglieder zum Abgeordnetenhaus ihr Amt angetreten haben“, gemäß

$12 \mathrm{~S}$. oben unter III.

13 Vergleiche die Meldung der Siam Times vom 19. 2. 69 nach der „unabhängige* Abgeordnete Prapat als Ministerpräsident vorschlugen.

14 So der jetzige Verkehrsminister Luftmarschall Thawi Chullasapaya in Siam Rath vom 19. 2. 69.

15 Auf sie bezog sich wohl die Außerung Prapats, daß nverschiedene rote Kandidaten, die eine große Summe Geldes im Wahlkampf ausgegeben hätten, gewählt worden seien ${ }^{\star}$, in Bangkok News vom 14.2 . 69.

16 Siam Rath, Prachathipatai, Phim Thai u. a. vom 13. 2. 69

17 Chao Thai, Siam Rath u. a. vom 15. 2. 69, item vom 16. 2. 69. 
Artikel 143 Abs. II muß jedoch „der aus dem Amt scheidende Ministerrat zur Ausführung der Geschäfte so lange im Amt bleiben, bis ein neuer Ministerrat ernannt worden ist".

Am 7. März d. J. wurde Feldmarschall Thanom Kitikačon auf Vorschlag des Präsidenten des Senats, Oberst Worakan, vom König wieder zum Ministerpräsidenten ernannt. Kitikačon tritt somit seine dritte Amtszeit als Ministerpräsident an. Am 10. März legte der Premier die Kabinettsliste vor, die vom König durch Ernennung der vorgeschlagenen zwölf Minister und 13 stellvertretenden Minister gebilligt wurde.

Sämtliche Regierungsmitglieder gehören der Saha Pracha Thai an oder stehen ihr nahe.

Die wichtigsten Ressorts wie die des Äußeren, der Verteidigung und der Finanzen blieben in derselben Hand wie vor der Wahl. Zur allgemeinen Überraschung behielt auch General Prapat sein Portefeuille, das Innenministerium, und wurde gleichzeitig 1. stellvertretender Ministerpräsident. In den Ruhestand traten die sechs Senioren des vorigen Kabinetts, darunter Prinz Wan Waithyakon, der bisherige 1. stellvertretende Ministerpräsident und ehemalige Präsident der Vollversammlung der Vereinten Nationen, sowie Momluong Pin Malakun, der langjährige Erziehungsminister.

Ein Teil des ehrgeizigen militärischen Nachwuchses mußte sich mit Ministerposten von minderer Bedeutung zufrieden geben. Der Generalstabschef, Luftmarschall Thawi, bisher gleichzeitig stellvertretender Verteidigungsminister, übernahm das Verkehrsministerium statt des von ihm angestrebten Verteidigungsministeriums. Letzteres blieb in der Hand des Premiers. Mehr Einfluß wurde dem wiederernannten Entwicklungsminister Pot Sarasin, einem der fähigsten "Technokraten" des Kabinetts, durch Ernennung zum 2. stellvertretenden Ministerpräsidenten eingeräumt.

VI. Unmittelbar nach den Wahlen traten verschiedene Abgeordnete der Opposition mit der Forderung nach Änderung der Verfassung hervor ${ }^{18}$, um diese "demokratischer zu gestalten“. Abgeordnete der regierenden Saha Pracha Thai schlossen sich dieser Forderung a ${ }^{19}$. Einzelheiten der beabsichtigten Anderung wurden noch nicht veröffentlicht. Man sollte diese Bestrebungen als ein ermutigendes Zeichen für die weitere Entwicklung Thailands ansehen.

Klaus Wenk

18 Seni Pramot in Siam Rath u. a. am 13. 2. 69; andere Abgeordnete in Phim Thai vom 14. 2. 69, Siam

Rath vom 17. 2. 69, Chau Thai vom 18. 2. 69 u. a.

19 Z. B. in Prachathipatai vom 16. 2. 69. 